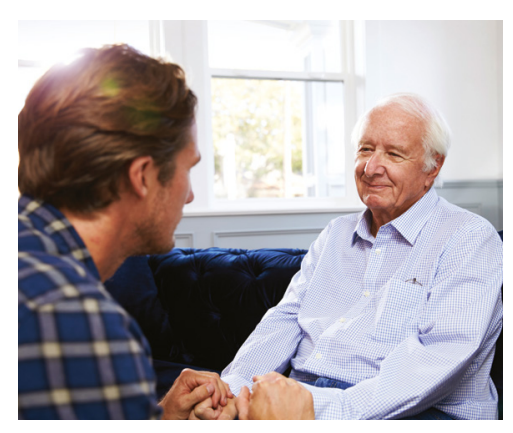

CPD

\section{Dimity Pond}

\section{Background}

Dementia is an increasingly prevalent condition, currently affecting over 400,000 Australians, and this is expected to rise to over one million by 2056. Diagnosis of dementia is a clinical one, as there is no single well-defined blood test, imaging or cognitive function test that validly diagnoses dementia. There are also many causes of cognitive impairment other than dementia, which need to be identified or excluded. General practitioners (GPs) are on the frontline of presentation and will therefore play an increasingly important role in identification and management.

\section{Objectives}

This article reviews what the GP can do in relation to office-based assessment for cognitive impairment.

\section{Discussion}

Dementia is an increasingly common condition, and it is important that GPs become familiar with its assessment and management in order to optimise access to care services, advance care planning and management of other conditions for their patients.

\title{
Office-based assessment of cognitive impairment
}

DEMENTIA IS A CONDITION with variable manifestations, affecting cognition, behaviour and the person's ability to perform activities of daily living. Over 46 million people live with dementia worldwide. This number is expected to increase to 131 million by 2050 because of the increasing longevity of the world population. ${ }^{1}$ Australia is no exception, with population numbers living with dementia expected to exceed one million by $2056 .^{2}$

What, then, is the role of the general practitioner (GP)? More than a decade ago, many GPs expected to refer their few incident cases of dementia to other specialists for investigation and diagnosis. However, as the numbers of people developing dementia increase, specialist referral is becoming less feasible economically and logistically, and so is now recommended only for those who wish to take anti-dementia medication, for complex cases where the diagnosis is unclear, or for those requesting to see another specialist. Some patients may be interested in referral to a centre conducting clinical trials of potential new treatments. However, some GPs may still prefer to refer cases for confirmation of the diagnosis. This article will discuss the steps for GPs to assess cognitive impairment in the case-finding group (ie those at moderate risk of dementia [Box 1]) in order to minimise visits and provide the specialist with a rationale for their decision-making.

\section{Why assess cognitive impairment?}

Before proceeding with investigation of cognitive impairment, it is worth asking whether the potential benefits of this assessment are likely to outweigh possible harms. Patients themselves have a range of views about this, and a large minority are not keen to be diagnosed with dementia. ${ }^{3}$ Indeed, the diagnosis is a grim one of a terminal illness with progressive deterioration. However, as with other terminal illnesses, it may be helpful for a person to know what is happening: while they still have capacity, they can complete their advance care planning (will, appointment of health and financial spokespeople, advance care directive), fulfil 'bucket list' plans and perhaps access anti-dementia medications and implement diet and exercise interventions to slow progression. Family and other carers should be educated about the implications of the diagnosis, as this aids understanding of prognosis and facilitates access to social care services. Once cognitive impairment is identified, the GP should also tailor management of comorbidities, putting in more structure and reminders, perhaps with the assistance of a care plan and the practice nurse.

If a diagnosis is made, whether of dementia or a milder degree of cognitive impairment that may progress to dementia, there is mounting evidence for secondary prevention that can slow the progression of the condition. For 
the GP, this includes full attention to cardiovascular risk factors and advice about diet, physical activity and social and cognitive engagement. ${ }^{4}$

\section{Who to assess}

The Royal Australian College of General Practitioners (RACGP) Guidelines for preventive activities in general practice 9 th edition (Red Book) does not suggest that GPs screen a whole population (eg those aged 75 years and over) for dementia, ${ }^{4}$ as there is insufficient evidence of benefit from this. ${ }^{5}$ The recently released National Health and Medical Research Council (NHMRC) Clinical practice guidelines and principles of care for people with dementia, known hereafter as the 'NHMRC Dementia Guidelines', 6 also do not recommend screening. Given the lack of a curative treatment, the high rates of false positives in the primary care population when using a cognitive function test alone, and the possible harms, including severe anxiety and loss of autonomy produced by a positive result, this is understandable. However, 'case finding' is promoted for those at moderate risk of dementia (Box 1). It should always be remembered that dementia can affect any age group, although it is more common as age progresses.

Box 1. Factors associated with moderate risk of dementia* 4,11

- Symptoms of dementia

- Increasing age (risk increases with age)

- Family history of Alzheimer's disease

- History of repeated head trauma

- Down syndrome

- Elevated cardiovascular risk (eg heart disease, stroke, hypertension, obesity, diabetes, elevated homocysteine, elevated cholesterol, smoking, sedentary lifestyle)

- Depression or a history of depression

- Low levels of education

- Identifying as an Aboriginal or Torres Strait Islander person

- Hearing impairment ${ }^{11}$

*The presence of one or more of these factors places a person at moderate risk of dementia and warrants case finding for cognitive impairment
The at-risk symptoms referred to in Box 1 are often 'soft' symptoms such as memory complaints or decline in thinking, planning or organising (eg inability to manage medications, missed appointments) or reduced emotional control (eg aggression towards staff and family members). Along with the other risk factors mentioned in Box 1, these symptoms, including relevant comments about them from staff or family members, should alert the GP to the need for further cognitive assessment, as people living with dementia may lack insight into their own deteriorating function.

\section{What assessments are required?}

Office-based assessment of cognitive impairment aims first to identify cognitive problems in those at moderate risk of dementia (Box 1) and then distinguish dementia from other common causes of cognitive impairment - the three 'Ds': delirium, depression and drug (medication) effects.

\section{Identifying a decline in cognitive function}

There are many screening scales for cognitive function. The RACGP Red Book (Table 5.5.2) ${ }^{4}$ recommends the Standardised Mini-Mental State Examination (replacing the Mini-Mental State Examination because of copyright issues), the General Practitioner Assessment of Cognition and the clock drawing test. For culturally and linguistically diverse communities, the Rowland Universal Dementia Assessment Scale is recommended, and for Aboriginal and Torres Strait Islander people, the Kimberley Indigenous Cognitive Assessment (KICA) tool. The NHMRC Dementia Guidelines suggest the KICACog or KICA-Screen for Aboriginal and Torres Strait Islander peoples in remote areas, and the modified KICA for urban and rural Aboriginal and Torres Strait Islander populations when illiteracy, language or cultural considerations deem it appropriate (Recommendations 39 and 40). ${ }^{6}$

None of these scales makes a diagnosis of dementia. A person with good education may score very well on these scales and still have dementia. A person who is poorly educated may score below the cut point and not have dementia. Many other issues affect the score, including: vision and hearing problems, psychiatric illness, anxiety, depression, being unwell on the day, cultural and linguistic diversity, neurological problems, manual dexterity for questions requiring use of a paper and pencil, and practice effects. The scales are simply a guide to cognitive function and need to be taken in context. Observing a deterioration over 6-12 months is a more reliable indicator of dementia than a single assessment. GPs should always review previous performance when they review scale marks. It is also helpful to look at the detailed marks if the scale had been administered by the practice nurse and not the GP themselves, to get an impression of where deficits lie. Ensure the practice nurse is familiar with the need to ask the questions exactly as worded and score them exactly as instructed. An interview with an informant who reports deterioration is also helpful.

\section{Excluding depression}

Depression is a well-known cause of poor cognitive function. Depression in older people may present in different ways compared with depression in younger people, with more apathy and social withdrawal rather than overt expressions of distress. The Geriatric Depression Scale $^{7}$ is useful for identifying depression in this age group, and it is often available on practice software.

A frequent reason for referral to specialist practice is the difficulty in distinguishing depression from dementia. Indeed, these two conditions often co-exist.

\section{Excluding delirium}

It is important to consider physical comorbidities, both acute and chronic, when assessing cognitive function. A physical examination should be conducted. Hypoxia in a person with chronic obstructive pulmonary disease, sleep apnoea, chronic hyperglycaemia, an acute chest infection or even constipation may impair cognitive function, and these conditions should be identified. 
A urinalysis should be performed to exclude active infection, glycosuria and renal disease.

A battery of blood tests is suggested (Box 2) to exclude underlying causes of cognitive impairment not mentioned above, including B12 and/or folate deficiency and thyroid disease, as well as other blood indicators of active disease. Syphilis and human immunodeficiency virus serology may be done if a history suggests these conditions as a possibility. A computed tomography scan is often recommended to exclude a brain tumour or other brain pathology that may mimic dementia. There is as yet no reliable imaging to rule in dementia.

\section{Excluding medication side effects}

Medications, particularly anticholinergic and psychotropic agents, may affect cognition. While most GPs know that anticholinergic agents cause dry mouth and blurred vision, many are not aware of their profound effect on cognition, particularly in older persons. Urinary antispasmodics are frequently a culprit in this age group. Many other common medications have low-level anticholinergic activity, but these may be additive to affect the ageing brain. ${ }^{8} \mathrm{~A}$ pharmacy review with a particular request to measure anticholinergic 'load', which numerically adds the anticholinergic burden across multiple medications, may assist and is remunerated under the Medicare Benefits Schedule. Psychotropic medications, including opioids and other medications for pain, should be used for a limited duration and withdrawn as soon as

\section{Box 2. Basic dementia screen to check for other causes of cognitive impairment $^{6}$}

- Full blood examination

- Biochemistry tests (including electrolytes, calcium, glucose, and renal and liver function)

- Thyroid function tests

- Serum vitamin B12 and folate levels

- Brain computed tomography

- Syphilis and human immunodeficiency virus serology if indicated possible. The GP should consider a gradual reduction in medication (deprescribing) in all older people, ${ }^{9}$ but particularly those with cognitive impairment.

\section{Functional capacity}

The diagnosis cannot be made without evidence of impaired functional capacity (ie reduced ability to perform activities of daily living). A number of scales exist. The Older Americans Resources and Services - Instrumental Activities of Daily Living Scale ${ }^{10}$ has been shown to be a significant predictor of service usage and may guide the decision for referral to care services. A significant component of functional assessment is the assessment of risk, including the risk of driving and of accidents in the home. Referral (eg for driving assessment) may help. Finding the balance between autonomy and safety is a difficult one and requires ongoing monitoring of functional capacity.

\section{What to tell the patient}

While GPs will not necessarily know the diagnosis at the outset of these office tests, they will have a better idea after completion of the tests. The patient may wish to know why these tests are being recommended or why a subsequent referral is being made. A suggested approach is 'I cannot exclude dementia from this examination, and would like to do some further testing for this. Is this OK?'

Once diagnosed, the NHMRC Dementia Guidelines state that a person has the 'right to know their diagnosis and the right not to know their diagnosis' (Principle 47), ${ }^{6}$ but stops short of indicating how to operationalise this recommendation in practice. A gradual and individualised approach is suggested (Principle 46) - for example, 'These problems/results suggest that you may have a problem with brain functioning: would you like to know more?'

\section{Dementia prevention}

While there is currently no cure for dementia, there is growing evidence for prevention strategies, both primary (ie before the disease is diagnosed) and secondary (ie to ameliorate symptoms or slow decline after diagnosis). The RACGP Red Book lists those with a good evidence base, including attention to cardiovascular risk factors, smoking cessation, dietary changes, exercise, social engagement and cognitive training. ${ }^{4}$

\section{Conclusion}

This brief article discusses office-based tests for cognitive impairment in the light of the demographic transition in the general practice patient population, which necessitates that GPs take a bigger role in diagnosing dementia. While the office tests suggested here may make the diagnosis clear in some cases, in other cases it will prove difficult to disentangle the effects of depression, physical illness and medication side effects from dementia itself. In such cases, the GP should refer to a specialist practice where possible. Specialists will prefer that the tests recommended here are done in advance of their seeing the patient, and this will speed up assessment and result in less anxiety and cost for the patient and the system overall. Once the diagnosis is made, the GP has an important role in ongoing management of the patient and, if possible, their carer, for this chronic deteriorating condition.

\section{Author}

Dimity Pond BA, MBBS, FRACGP, PhD, Head, Discipline of General Practice, School of Medicine and Public Health, Faculty of Health, University of Newcastle. Dimity.Pond@newcastle.edu.au Competing interests: None.

Funding: None.

Provenance and peer review: Commissioned, externally peer reviewed.

\section{References}

1. Prince M, Wimo A, Guerchet $M$, et al. World Alzheimer report 2015: The global impact of dementia. An analysis of prevalence, incidence, cost and trends. London: Alzheimer's Disease International, 2015.

2. Brown L, Hansnata E, Anh La H. Economic cost of dementia in Australia 2016-2056. NATSEM at the Institute for Governance and Policy Analysis, University of Canberra. Report prepared for Alzheimers Australia. Canberra: University of Canberra, 2017. Available at: https://staff. dementia.org.au/files/NATIONAL/documents/ The-economic-cost-of-dementia-in-Australia2016-to-2056.pdf [Accessed 15 April 2018]. 
3. Magin P, Juratowitch L, Dunbabin J, at al. Attitudes to Alzheimer's disease testing of Australian general practice patients: A cross-sectional questionnaire-based study. Int J Geriatr Psychiatry 2016;31(4):361-66. doi:10.1002/gps.4335.

4. The Royal Australian College of General Practitioners. Guidelines for preventive activities in general practice. 9th edn. Melbourne: RACGP, 2016.

5. Mate KE, Magin PJ, Brodaty $\mathrm{H}$, et al. An evaluation of the additional benefit of population screening for dementia beyond a passive case-finding approach. Int J Geriatr Psychiatry 2017;32(3):316-23. doi:10.1002/gps.4466.

6. Guideline Adaptation Committee. Clinical practice guidelines and principles of care for people with dementia. Sydney: Guideline Adaptation Committee, 2016.

7. Sheikh JI, Yesavage J A. Geriatric Depression Scale (GDS): Recent evidence and development of a shorter version. Clin Gerontol 1986;5(102):165-73. Available at http://dementiakt.com.au/doms/ domains/behaviour/geriatric-depression-scale/ [Accessed 16 April 2018].
8. Magin PJ, Morgan S, Tapley A, et al. Anticholinergic medicines in an older primary care population: A cross-sectional analysis of medicines' levels of anticholinergic activity and clinical indications. J Clin Pharm Ther 2016;41(5):486-92.

9. Scott I, Hilmer S, Reeve S, et al. Reducing inappropriate polypharmacy: The process of deprescribing. JAMA Intern Med 2015:175(5):827-34. doi:10.1001/ jamainternmed.2015.0324.

10. Fillenbaum GG. Multidimensional functional assessment of older adults: The Duke Older Americans Resources and Services procedures. Hillsdale, NJ: Lawrence Erlbaum Associates Inc, 1988. Available at http://dementiakt.com.au/ doms/domains/function/oars-iadl/ [Accessed 16 April 2018]

11. Livingstone G, Sommerlad A, Orgeta V, et al. Dementia prevention, intervention, and care. Lancet 2017;390(10113):2673-34. doi: 10.1016/ S0140-6736(17)31363-6 [Accessed 6 July 2018]. 